Tuberc Respir Dis 2012;72:481-485

Copyright(C)2012. The Korean Academy of Tuberculosis and Respiratory Diseases. All rights reserved.

\title{
Factors Determining the Timing of Tracheostomy in Medical ICU of a Tertiary Referral Hospital
}

\author{
Young Sik Park, M.D., Jinwoo Lee, M.D., Sang-Min Lee, M.D., Ph.D., Jae-Joon Yim, M.D., Ph.D., Young \\ Whan Kim, M.D., Ph.D., Sung Koo Han, M.D., Ph.D., Chul-Gyu Yoo, M.D., Ph.D. \\ Division of Pulmonary and Critical Care Medicine, Department of Internal Medicine and Lung Institute, Seoul National University \\ College of Medicine, Seoul, Korea
}

\begin{abstract}
Background: Tracheostomy is a common procedure for patients requiring prolonged mechanical ventilation. However, the timing of tracheostomy is quite variable. This study was performed to find out the factors determining the timing of tracheostomy in medical intensive care unit (ICU).

Methods: Patients who were underwent tracheostomy between January 2008 and December 2009 in the medical ICU of Seoul National University Hospital were included in this retrospective study.

Results: Among the 59 patients, 36 (61.0\%) were male. Median Acute Physiology And Chronic Health Evaluation (APACHE) II scores and Sequential Organ Failure Assessment scores on the admission day were 28 and 7 , respectively. The decision of tracheostomy was made on 13 days, and tracheostomy was performed on 15 days after endotracheal intubation. Of the 59 patients, 21 patients received tracheostomy before 2 weeks (group I) and 38 were underwent after 2 weeks (group II). In univariate analysis, days until the decision to perform tracheostomy ( 8 vs. $14.5, \mathrm{p}<0.001$ ), days before tracheostomy (10 vs. $18, \mathrm{p}<0.001)$, time delay for tracheostomy $(2.1 \mathrm{vs} .3 .0$, $\mathrm{p}<0.001)$, cardiopulmonary resuscitation $(19.0 \%$ vs. 2.6\%, $\mathrm{p}=0.049)$, existence of neurologic problem $(38.1 \%$ vs. 7.9\%, p=0.042), APACHE II scores (24 vs. 30, p=0.002), and $\mathrm{PaO}_{2} / \mathrm{FiO}_{2}<300 \mathrm{~mm} \mathrm{Hg}(61.9 \%$ vs. 91.1\%, $\mathrm{p}=0.011$ ) were different between the two groups. In multivariate analysis, APACHE II scores $\geq 20$ (odds ratio [OR], 12.44; $95 \%$ confidence interval [CI], 1.14 136.19; $\mathrm{p}=0.039$ ) and time delay for tracheostomy (OR, 1.97; 95\% CI, 1.11 3.55; $\mathrm{p}=0.020)$ were significantly associated with tracheostomy after 2 weeks.
\end{abstract}

Conclusion: APACHE II scores $\geq 20$ and time delay for tracheostomy were associated with tracheostomy after 2 weeks.

Key Words: APACHE; Intensive Care Units; Time; Tracheostomy

\section{Introduction}

Tracheostomy is a common surgical procedure in the intensive care unit (ICU) for patients requiring prolonged mechanical ventilation. Theoretically, a tracheostomy has some advantages over a translaryngeal endotracheal tube for respiratory physiology, nursing care,

Address for correspondence: Chul-Gyu Yoo, M.D., Ph.D. Division of Pulmonary and Critical Care Medicine, Department of Internal Medicine and Lung Institute, Seoul National University College of Medicine, 101, Daehak-ro, Jongno-gu, Seoul 110-744, Korea

Phone: 82-2-2072-3760, Fax: 82-2-762-9662

E-mail: cgyoo@snu.ac.kr

Received: Mar. 29, 2012

Revised: Apr. 13, 2012

Accepted: May 10, 2012 and psychology ${ }^{1,2}$. Although some beneficial effects of early tracheostomy have been reported ${ }^{3}$, recent large studies were unable to show a reduced incidence of ventilator-associated pneumonia (VAP) and ICU mortality in patients with an early tracheostomy compared to those with a late tracheostomy ${ }^{4,5}$. Despite that it is a common procedure, the optimal time for a tracheostomy in the ICU is not yet clearly defined ${ }^{1}$. Furthermore, the selection of patients and timing of the decision for a tracheostomy are subjective, as no reliable tests have been established to predict the need for prolonged ventilation.

Intubation for 2 weeks is considered safe with the use of a low-pressure and high-volume endotracheal tube 
cuff $^{6}$. According to nationwide surveys, a tracheostomy was performed within 14 days in 90\% of German ICUs ${ }^{7}$ and within 21 days in $68 \%$ of French ICUs ${ }^{8}$. The criterion for an early tracheostomy in clinical trials was defined from as early as 48 hours $^{3}$ to as late as 8 days $^{5}$.

Traditionally, 2 weeks was the time limit to make the decision for a tracheostomy in all patients requiring prolonged mechanical ventilation. The timing of a tracheostomy is diverse, and delaying a tracheostomy for more than 2 weeks is not uncommon in the ICU, but the reasons for such a delayed tracheostomy have not been investigated. This study was performed to determine the exact timing of a tracheostomy and factors influencing tracheostomy timing in the medical ICU of a tertiary referral hospital in Korea.

\section{Materials and Methods}

\section{Study population}

Patients who were admitted to the medical ICU (22 beds) of Seoul National University Hospital from January 2008 to December 2009 were recruited. Inclusion criteria were age $>18$ years, mechanical ventilation with translaryngeal intubation and first tracheostomy. Exclusion criteria were postoperative patients, multiple separate ICU admissions during the same hospital stay and uncontrolled or haematological malignancy. This protocol (H-1006-141-322) was approved by the Seoul National University Hospital Institutional Review Board.

\section{Assessment of organ failure}

Organ failure was defined as a Sequential Organ Failure Assessment (SOFA) score ${ }^{9} \geq 2$ on each item, as follows: $\mathrm{PaO}_{2} / \mathrm{FiO}_{2}<300 \mathrm{~mm} \mathrm{Hg}$, the use of inotropics or vasopressors, platelets $<100,000 / \mathrm{mm}^{3}$, bilirubin $\geq 2.0$ $\mathrm{mg} / \mathrm{dL}$, creatinine $\geq 2.0 \mathrm{mg} / \mathrm{dL}$ and Glasgow Coma Scale $\leq 12$. The development of organ failure was defined as the existence of the above criteria from ICU admission to the day of the tracheostomy.

\section{Statistical analysis}

p-values were calculated using the $\chi^{2}$ test or Fisher's exact test for categorical variables and the MannWhitney U-test for continuous variables. Odds ratios (ORs) were calculated from the logistic regression model in the multivariate analysis. A $p<0.05$ was considered to indicate statistical significance. STATA version 11.0 (StataCorp., College Station, TX, USA) was used for the analysis.

\section{Results}

Fifty-nine patients were included in the study (Table 1). The median age of the patients was 68 years (range, $30 \sim 88$ years), and male gender was predominant (61.0\%). The common causes of medical ICU admission were respiratory failure $(69.5 \%)$ and neurological problems (18.6\%), sepsis (10.2\%) and cardiac problems (1.7\%). Cardiopulmonary resuscitation (CPR) was performed in five of 59 patients prior to medical ICU admission. Median Acute Physiology And Chronic Health Evaluation (APACHE) II score ${ }^{10}$ and SOFA score on ICU admission day were 28 (range, $12 \sim 40$ ) and 7 (range, $1 \sim 17$ ), respectively. The patients were given mechanical ventilation support for 23 days (range, 6 95 days) and remained in the medical ICU for 28 days (range, $7 \sim 95$ days). Under mechanical ventilation support, the decision to perform a tracheostomy was performed on day 13 (range, $2 \sim 28$ days) and the actual bedside operation was done on day 15 (range, 5 31 days). The time delay for tracheostomy was 3 days (range, $0 \sim 21$ days).

Patients were grouped according to the day of tracheostomy, i.e., whether it was performed before or after 2 weeks. Among the 59 patients, 21 (35.6\%) received a tracheostomy before 2 weeks (group I), and 38 (64.4\%) received one after 2 weeks (group II) (Table 2). In the univariate analysis, the decision and operation time of the tracheostomy were significantly different between the two groups. The time delay for tracheostomy was also different between group I and group II. Compared with group II, group I showed more frequent CPR (19\% vs. 2.6\%, $\mathrm{p}=0.049)$, more common neurological problems $(38.1 \%$ vs. $7.9 \%, \mathrm{p}=0.042)$ and lower

\section{2}


Table 1. Baseline characteristics of the 59 participants

\begin{tabular}{|c|c|}
\hline Variable & No. $(\%)(n=59)$ \\
\hline Age, yr & $68(30 \sim 88)$ \\
\hline Male & $36(61.0)$ \\
\hline Cardiopulmonary resuscitation & $5(8.5)$ \\
\hline \multicolumn{2}{|l|}{ Reason for ICU admission } \\
\hline Respiratory failure & $41(69.5)$ \\
\hline Neurologic problem & $11(18.6)$ \\
\hline Sepsis & $6(10.2)$ \\
\hline Cardiologic problem & $1(1.7)$ \\
\hline APACHE \| scores & $28(12 \sim 40)$ \\
\hline \multicolumn{2}{|l|}{ SOFA scores } \\
\hline ICU admission day & $7(1 \sim 17)$ \\
\hline Tracheostomy day & $6(2 \sim 18)$ \\
\hline Mechanical ventilation days, day & $23(6 \sim 95)$ \\
\hline ICU days, day & $28(7 \sim 95)$ \\
\hline Hospital days, day & $55(11 \sim 731)$ \\
\hline \multicolumn{2}{|l|}{$\begin{array}{l}\text { Organ failure assessment from ICU } \\
\text { admission day to tracheostomy day }\end{array}$} \\
\hline $\mathrm{PaO}_{2} / \mathrm{FiO}_{2}<300 \mathrm{~mm} \mathrm{Hg}$ & $48(81.4)$ \\
\hline Inotropics or vasopressor use & $35(59.3)$ \\
\hline Platelet $<100,000 / \mathrm{mm}^{3}$ & $18(30.5)$ \\
\hline Bilirubin $\geq 2.0 \mathrm{mg} / \mathrm{dL}$ & $8(13.6)$ \\
\hline Creatinine $\geq 2.0 \mathrm{mg} / \mathrm{dL}$ & $13(22.0)$ \\
\hline GCS score $\leq 12$ & $16(27.1)$ \\
\hline Time for tracheostomy decision, day & $13(2 \sim 28)$ \\
\hline Time for tracheostomy, day & $15(5 \sim 31)$ \\
\hline Time delay for tracheostomy, day & $3(0 \sim 21)$ \\
\hline In ICU mortality & $17(28.8)$ \\
\hline \multicolumn{2}{|l|}{ Discharge } \\
\hline Home & $17(28.8)$ \\
\hline Transfer to other hospital & $22(37.3)$ \\
\hline Death & $20(33.9)$ \\
\hline
\end{tabular}

Values are presented as number (\%) or median (range). ICU: intensive care unit; APACHE: acute physiology and chronic health evaluation; SOFA: sequential organ failure assessment; GCS: Glasgow Coma Score.

APACHE II scores (24 vs. 30, $\mathrm{p}=0.002$ ). In the assessment of organ failure, group II patients showed a higher frequency of $\mathrm{PaO}_{2} / \mathrm{FiO}_{2}<300 \mathrm{~mm} \mathrm{Hg}(61.9 \%$ vs. 92.1\%, $\mathrm{p}=0.011$ ). Table 3 shows the multivariate analysis for a tracheostomy after 2 weeks. Our data revealed that higher APACHE II score ( $\geq 20$ ) (OR, 12.44; 95\% confidence interval [CI], 1.14 136.19; $\mathrm{p}=0.039)$ and time delay for tracheostomy (OR, 1.97; 95\% CI, 1.11 3.55, $\mathrm{p}=0.020$ ) were independent significant factors for performing a tracheostomy after 2 weeks.

\section{Discussion}

Early prediction of patients who will eventually need a tracheostomy is very difficult. The time for a tracheostomy varies, and the optimal time has not been established. Dunham and LaMonica ${ }^{6}$ reported that endotracheal intubation for up to 2 weeks is not associated with increased complications related to translaryngeal intubation. The consensus conference recommends performing a tracheostomy after 3 weeks of translaryngeal intubation $^{11}$. Compared to this relatively late tracheostomy, recent studies have shown a clinical benefit of an early tracheostomy ${ }^{4,5,12,13}$. Some studies have shown that early tracheostomy was related with early weaning from mechanical ventilation; however, a large randomised controlled trial failed to demonstrate a reduction in VAP and mortality through the use of an early tracheostomy ${ }^{5}$.

According to our results, the median decision time for a tracheostomy was 13 days, and a tracheostomy was performed at day 15 after translaryngeal intubation. Compared to recent clinical trials, this could be considered a late tracheostomy. Some possible explanations for this include the following. First, the standardised tracheostomy protocol and early-tracheostomy decision algorithm may be helpful. The objective decision algorithm for a tracheostomy predicts prolonged ventilation more effectively ${ }^{14}$, whereas the standardised protocol for a tracheostomy may minimise clinical variation ${ }^{15}$. Second, the time gap between decision-making and the actual operation of the tracheostomy was 3 days. Furthermore the time delay for tracheostomy was significantly different between group I and group II. This meant that 3 days were needed for interdepartmental consultation with otolaryngologists and surgical preparation for the tracheostomy and as the time delayed more, the actual tracheostomy was performed lately. As the standard method for a tracheostomy was a bedside surgical procedure at that time in our hospital, the time gap was inevitable. After recently introducing percutaneous dilatational tracheostomy in our medical ICU, we have been able to successfully shorten the gap. 
YS Park et al: Factors determining the timing of tracheostomy in medical ICU

Table 2. Univariate analysis of tracheostomy before and after 2 weeks

\begin{tabular}{|c|c|c|c|}
\hline Variables & $<2$ weeks $(n=21)$ & $\geq 2$ weeks $(n=38)$ & p-value \\
\hline Time for tracheostomy decision & $8(2 \sim 13)$ & 14.5 (4 28) & $<0.001$ \\
\hline Time for tracheostomy & $10(5 \sim 13)$ & $18(14 \sim 31)$ & $<0.001$ \\
\hline Time delay for tracheostomy & $2.1(0 \sim 5)$ & $3.0(0 \sim 21)$ & $<0.001$ \\
\hline Age, yr & 67 (33 81) & 69 (30 88) & 0.640 \\
\hline Male & $15(71.4)$ & $21(55.3)$ & 0.223 \\
\hline Cardiopulmonary resuscitation & $4(19.0)$ & $1(2.6)$ & 0.049 \\
\hline \multicolumn{4}{|l|}{ Reason for ICU admission } \\
\hline Respiratory failure & $11(52.4)$ & $30(78.9)$ & 0.355 \\
\hline Neurologic problem & $8(38.1)$ & $3(7.9)$ & 0.042 \\
\hline Sepsis & $2(9.5)$ & $4(10.5)$ & 1.000 \\
\hline Cardiologic problem & $0(0)$ & $1(2.6)$ & 1.000 \\
\hline APACHE \| scores & $24(12 \sim 37)$ & $30(12 \sim 40)$ & 0.002 \\
\hline \multicolumn{4}{|l|}{ SOFA scores } \\
\hline ICU admission day & $7(1 \sim 17)$ & $7(3 \sim 15)$ & 0.981 \\
\hline Tracheostomy day & $5(2 \sim 15)$ & $6(2 \sim 18)$ & 0.556 \\
\hline Mechanical ventilation days, day & 17 (6 88) & 32 (9 95) & 0.003 \\
\hline ICU days, day & 20 (7 91) & $33(15 \sim 95)$ & 0.004 \\
\hline Hospital days, day & $42(11 \sim 264)$ & $59.5(21 \sim 731)$ & 0.046 \\
\hline \multicolumn{4}{|c|}{$\begin{array}{l}\text { Organ failure assessment from ICU admission } \\
\text { day to tracheostomy day }\end{array}$} \\
\hline $\mathrm{PaO}_{2} / \mathrm{FiO}_{2}<300 \mathrm{~mm} \mathrm{Hg}$ & $13(61.9)$ & $35(92.1)$ & 0.011 \\
\hline Inotropics or vasopressor use & $11(52.4)$ & $24(63.2)$ & 0.420 \\
\hline Platelet $<100,000 / \mathrm{mm}^{3}$ & $4(19.0)$ & $14(36.8)$ & 0.155 \\
\hline Bilirubin $\geq 2.0 \mathrm{mg} / \mathrm{dL}$ & $2(9.5)$ & $6(15.8)$ & 0.699 \\
\hline Creatinine $\geq 2.0 \mathrm{mg} / \mathrm{dL}$ & $3(14.3)$ & $10(26.3)$ & 0.344 \\
\hline GCS score $\leq 12$ & $8(38.1)$ & $8(21.1)$ & 0.159 \\
\hline Sum of organ failure no. & $2(0 \sim 5)$ & $2(0 \sim 5)$ & 0.115 \\
\hline In ICU mortality & $6(28.6)$ & $11(28.9)$ & 0.976 \\
\hline \multicolumn{4}{|l|}{ Discharge } \\
\hline Home & $5(23.8)$ & $12(31.6)$ & 0.636 \\
\hline Transfer to other hospital & $9(42.9)$ & $13(34.2)$ & 0.659 \\
\hline Death & $7(33.3)$ & $13(34.2)$ & 0.946 \\
\hline
\end{tabular}

Values are presented as number (\%) or median (range).

ICU: intensive care unit; APACHE: acute physiology and chronic health evaluation; SOFA: sequential organ failure assessment; GCS: Glasgow Coma Score.

Table 3. Multivariate analysis of tracheostomy after 2 weeks

\begin{tabular}{lcc}
\hline \multicolumn{1}{c}{ Variables } & OR $(95 \% \mathrm{Cl})$ & p-value \\
\hline Age & $1.00(0.93 \sim 1.07)$ & 0.888 \\
Gender (female) & $0.23(0.03 \sim 1.71)$ & 0.150 \\
Cardiopulmonary resuscitation & $0.92(0.004 \sim 2.11)$ & 0.135 \\
Neurologic problem & $0.29(0.02 \sim 4.10)$ & 0.362 \\
APACHE II scores $\geq 20$ (ICU admission day) & $12.44(1.14 \sim 136.19)$ & 0.039 \\
SOFA scores (ICU admission day) & $0.91(0.68 \sim 1.20)$ & 0.490 \\
SOFA scores (tracheostomy day) & $0.93(0.73 \sim 1.17)$ & 0.518 \\
$\mathrm{PaO}_{2} /$ FiO 2 300 mm Hg & $6.25(0.22 \sim 179.79)$ & 0.285 \\
Time delay for tracheostomy, day & $1.97(1.11 \sim 3.55)$ & 0.020 \\
\hline
\end{tabular}

OR: odds ratio; Cl: confidence interval; APACHE: acute physiology and chronic health evaluation; ICU: intensive care unit; SOFA: sequential organ failure assessment. 
We found some similar patterns in the decision-making concerning tracheostomy. Most of the patients who were assessed with an irreversible brain injury (previous neurological problems or post $\mathrm{CPR}$ ) received an early $(<2$ weeks) tracheostomy (Table 2$)$, while patients who had higher APACHE II scores received a late $(\geq 2$ weeks) tracheostomy. If high mortality was expected on the day of medical ICU admission, the decision and procedure time for the tracheostomy were delayed. Also, a late tracheostomy was preferred in the event of respiratory failure $\left(\mathrm{PaO}_{2} / \mathrm{FiO}_{2}<300 \mathrm{~mm} \mathrm{Hg}\right)$.

Effects of a selection bias may exist due to the retrospective nature of this study. According to our results, the median time of tracheostomy was 15 days after endotracheal intubation. The patient must be alive at the time with tolerable operative conditions to receive a tracheostomy. If a patient showed unfavourable operative conditions, the tracheostomy might have be postponed. During these delays, the critically ill cases may have died before a tracheostomy. In other words, only those who survived longer in the medical ICU received a tracheostomy. Considering this selection bias, we did not compare the mortality according to the timing of the tracheostomy.

In conclusion, tracheostomy was performed a median of 15 days after endotracheal intubation in the medical ICU. Our data suggest that high APACHE II scores $(\geq 20)$ may influence the timing of a tracheostomy in a medical ICU. And the time gap caused by interdepartmental consultation for tracheostomy was another independent factor for delayed tracheostomy.

\section{References}

1. Holevar M, Dunham JC, Brautigan R, Clancy TV, Como JJ, Ebert JB, et al. Practice management guidelines for timing of tracheostomy: the EAST Practice Management Guidelines Work Group. J Trauma 2009;67:870-4.

2. Epstein SK. Anatomy and physiology of tracheostomy. Respir Care 2005;50:476-82.

3. Rumbak MJ, Newton M, Truncale T, Schwartz SW, Adams JW, Hazard PB. A prospective, randomized, study comparing early percutaneous dilational tracheotomy to prolonged translaryngeal intubation (delayed tracheotomy) in critically ill medical patients. Crit Care Med 2004;32:1689-94.

4. Zagli G, Linden M, Spina R, Bonizzoli M, Cianchi G, Anichini V, et al. Early tracheostomy in intensive care unit: a retrospective study of 506 cases of video-guided Ciaglia Blue Rhino tracheostomies. J Trauma 2010;68: 367-72.

5. Terragni PP, Antonelli M, Fumagalli R, Faggiano C, Berardino M, Pallavicini FB, et al. Early vs late tracheotomy for prevention of pneumonia in mechanically ventilated adult ICU patients: a randomized controlled trial. JAMA 2010;303:1483-9.

6. Dunham CM, LaMonica C. Prolonged tracheal intubation in the trauma patient. J Trauma 1984;24:120-4.

7. Kluge S, Baumann HJ, Maier C, Klose H, Meyer A, Nierhaus A, et al. Tracheostomy in the intensive care unit: a nationwide survey. Anesth Analg 2008;107:163943.

8. Blot F, Melot C; Commission d'Epidémiologie et de Recherche Clinique. Indications, timing, and techniques of tracheostomy in 152 French ICUs. Chest 2005;127: $1347-52$.

9. Vincent JL, Moreno R, Takala J, Willatts S, De Mendonça A, Bruining $\mathrm{H}$, et al. The SOFA (Sepsis-related Organ Failure Assessment) score to describe organ dysfunction/failure. On behalf of the Working Group on Sepsis-Related Problems of the European Society of Intensive Care Medicine. Intensive Care Med 1996;22:70710 .

10. Knaus WA, Draper EA, Wagner DP, Zimmerman JE. APACHE II: a severity of disease classification system. Crit Care Med 1985;13:818-29.

11. Plummer AL, Gracey DR. Consensus conference on artificial airways in patients receiving mechanical ventilation. Chest 1989;96:178-80.

12. Freeman BD, Borecki IB, Coopersmith CM, Buchman TG. Relationship between tracheostomy timing and duration of mechanical ventilation in critically ill patients. Crit Care Med 2005;33:2513-20.

13. Scales DC, Thiruchelvam D, Kiss A, Redelmeier DA. The effect of tracheostomy timing during critical illness on long-term survival. Crit Care Med 2008;36:2547-57.

14. Durbin CG Jr, Perkins MP, Moores LK. Should tracheostomy be performed as early as 72 hours in patients requiring prolonged mechanical ventilation? Respir Care 2010;55:76-87.

15. Freeman BD, Kennedy C, Robertson TE, Coopersmith CM, Schallom M, Sona C, et al. Tracheostomy protocol: experience with development and potential utility. Crit Care Med 2008;36:1742-8. 